\title{
Impact of Pulse Length on the accuracy of Defect Depth Measurements in Pulse Thermography
}

James Pierce

University of South Florida, Tampa

Nathan B. Crane

Brigham Young University - Provo, nbcrane@byu.edu

Follow this and additional works at: https://scholarsarchive.byu.edu/facpub

Part of the Other Mechanical Engineering Commons

\section{Original Publication Citation}

James Pierce and Nathan B. Crane, "Impact of pulse length on the accuracy of defect depth measurements in pulse thermography," Journal of Heat Transfer, v 141, n 4, April 1, 2019

\section{BYU ScholarsArchive Citation}

Pierce, James and Crane, Nathan B., "Impact of Pulse Length on the accuracy of Defect Depth Measurements in Pulse Thermography" (2019). Faculty Publications. 5342.

https://scholarsarchive.byu.edu/facpub/5342 


\title{
Impact of Pulse Length on the accuracy of Defect Depth Measurements in Pulse Thermography
}

\author{
James Pierce ${ }^{1}$ and Nathan B. Crane ${ }^{2}$ \\ ${ }^{1}$ University of South Florida, Tampa, Florida 33620 \\ ${ }^{2}$ Brigham Young University, Provo, UT 84602, nbcrane@byu.edu
}

\begin{abstract}
Pulse thermography is a nondestructive testing method in which an energy pulse is applied to a surface while the surface temperature evolution is measured to detect sub surface defects and estimate their depth. This nondestructive test method was developed on the assumption of instantaneous surface heating, but recent work has shown that relatively long pulses can be used to accurately determine defect depth in polymers. This paper examines the impact of varying input pulse length on the accuracy of defect depth quantification as a function of the material properties. Simulations using both thermoplastics and metals show that measurement error is dependent on a nondimensionalized pulse length. The simulation results agree with experimental results for 3D printed Acrylonitrile butadiene styrene (ABS) and polylactic acid (PLA) components. Analysis and experiments show that defects can be accurately detected with minor modification to the standard methods as long as the pulse ends before the characteristic defect signal is detected.
\end{abstract}

\section{Introduction}

Pulse Thermography (PT) is a form of infrared thermography for quantification of subsurface defects in materials. In this method a short pulse of energy is applied to the surface (typically by flash lamps, pulse length $\sim 2-10 \mathrm{~ms}$ ). The temperature of the surface is monitored via an infrared (IR) camera and the thermal response is used to detect defects and estimate their depth.

After the flash, one-dimensional (1D) heat conduction begins into the part. While some radiative and convective losses may be expected, these are generally neglected due to the small temperature change relative to ambient $\left(<5^{\circ} \mathrm{C}\right)$. Parker et al. [1] showed that the decay of the surface temperature follows the equation:

$$
T(t)=\frac{Q}{\rho C L}\left[1+2 \sum_{n=1}^{\infty} \exp \left(-\frac{n^{2} \pi^{2}}{L^{2}} \alpha t\right)\right]
$$

where $\mathrm{Q}$ is the input energy per unit area on the surface of the part, $\mathrm{C}$ is the specific heat capacity of the material, $\rho$ is the density of the part, $L$ is the thickness of the part, and $\alpha$ is the thermal diffusivity. The equation was derived with three major assumptions: no heat loss from the surfaces of the part as seen in Figure 1, the thermal input is an instantaneous pulse (Dirac pulse), and thus, there is a negligible internal temperature distribution immediately following the pulse. To meet the last two assumptions, previous work utilizing this method [2-7] have used flash lamps to thermally excite the surface of the specimens they were testing with 2-10 ms pulses, with most at the shorter end of the range. 
Where a defect such as foreign material, void, or a delamination is present within the part, the effective thermal resistance is much higher than over the sound (defect free) area of the part. This slows down the heat transfer within the part directly above the defective region, thus producing a thermal contrast on the surface relative to sound areas of the part. There are several methods that have been found to quantify the defect depth based on this thermal contrast on the surface, but the two most common methods are peak temperature contrast method and log second derivative method. The peak temperature contrast slope method derived by Ringermacher et al [3] utilizes the derivative of the temperature contrast between the sound area and the defective area over time. The time the peak of the derivative occurs can be directly correlated with the square of the defect depth as seen in equation 2 :

$$
t_{s}=\frac{3.64 L^{2}}{\pi^{2} \alpha}
$$

where $t_{s}$ is the peak time, and $L$ is the depth of the defect. The constant of 3.64, more specifically 3.6359 , is obtained from the derivative of the surface temperature difference equation of the defective and sound region (Sun et al. [6]). When the equation is evaluated in reference to a defect depth to part thickness ratio less than 0.5, it yields the constant 3.6359. Beyond that the constant must be adjusted to yield accurate defect depth quantification.

Shepherd et al. [5] developed the log second derivative method. They found that in the $\log$ scale the temperature decay on the surface is linear with a -0.5 slope. However, when a defect is present, the temperature decay in the log scale deviates from this -0.5 slope. The second derivative of the temperature decay produces a peak and the time this peak occurs can also be directly correlated with the square of the defect depth with equation 3 :

$$
t_{2}=\frac{L^{2}}{\pi \alpha}
$$

where $t_{2}$ is the peak time. The benefit of the log second derivative method over the peak temperature contrast method is that it does not require a reference sound area for calculation of the defect depth. Similar pulse length dependence is seen in both methods, but this paper will focus on the impact of a longer pulse on the depth estimation in the peak temperature contrast slope method.

A nondimensional measurement of the pulse length $(\tau)$ can be obtained by dividing the pulse length $\left(t_{p}\right)$ by the $t_{s}$ and $t_{2}$ values for the peak temperature contrast and log second derivative methods, respectively. The measured values of peak temperatures can be used or Equations 2 and 3 can be substituted to make predictions based on defect depths of interest. to get the following equations for the peak temperature contrast and $\log 2^{\text {nd }}$ derivative methods respectively:

$$
\tau_{s}=\frac{t_{p}}{t_{s}}=\frac{\pi^{2} \alpha t_{p}}{3.64 L^{2}}
$$



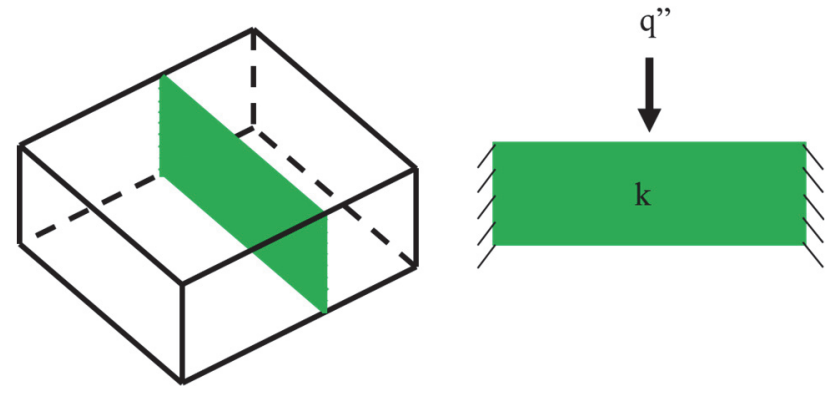

Figure 1 Schematic representation of the 1D boundary condition for PT. The green slice represents the simplified 1D heat transfer representation of the 3D specimen.

$$
\tau_{2}=\frac{t_{p}}{t_{2}}=\frac{\pi \alpha t_{p}}{L^{2}}
$$

In implementing these processes, it is important to know how the measurement error will be impacted by the pulse length for different combinations of material properties and defect depths. A "short" pulse will clearly be different for a material such as 316 stainless steel and ABS P400 with a thermal diffusivity approximately 50 times smaller than $316 \mathrm{SS}$. However, the pulse length requirements and their dependence on the material properties of the sample have not been characterized. Thus, the next sections will discuss the benefits of using a longer pulse and analyze how the material properties affect the accuracy of a calculated defect depth using different pulse lengths.

\section{Benefit of Longer Pulse}

Longer input pulses provide several measurement benefits. For example, a longer pulse allows a larger energy input into the system without having to change the power source or lamp. Higher energy dramatically increases the temperature change which reduces the sensitivity to measurement noise. With a $4 \mathrm{~kW}$ power source, the energy input is 50 times larger with a 100ms pulse than would be a $2 \mathrm{~ms}$ pulse, producing approximately a 50 times larger surface temperature contrast for the same material. For materials with large thermal diffusivities or shallow defects this may not be as critical as the temperature signal is large enough to overcome thermal noise. However, for materials with small thermal diffusivities and when analyzing deeper defects, the ability to simply adjust the pulse time to increase the energy allowing for a larger temperature signal is quite appealing. Additionally, longer pulses can be achieved with standard halogen or LED lighting without the expense of a high-power pulsed voltage source.

This ability to use a longer pulse is especially attractive in nontraditional applications to low thermal diffusivity materials such as polymers and powders. These applications are of particular interest in the additive manufacturing (AM) of metal components via powder processes. As shown by Masamune and Smith [8], thermal conductivity of powder beds is significantly smaller than the thermal conductivity of their bulk material counterpart. Thus, the thermal diffusivities are significantly smaller as well and modified testing parameters/equipment would be required for studying powderbed and green components produced by processes such as binder jetting [9]. These techniques would also be appropriate for studying other green powder parts 
produced by traditional metal and ceramic powder techniques and for polymer AM components. The longer pulse would be helpful for defect detection and improved accuracy.

Previous studies have already begun to look at the use of longer pulses up to essentially continuous heating for defect detection. Kim et al [10] showed that active thermography with a heating duration of 150 s could detect wall thinning in nuclear pipe components though no effort was made to quantify thickness changes. Recently Almond et al. [11] studied a new method of analytical quantification of defect depths using pulse lengths of 5s. Based on the estimated heat flux applied to the surface above a defective region, they could compare the experimental temperature contrast to predicted contrast and correlate that to a prescribed defect depth. Recently, Pierce and Crane [12] testing a low thermal diffusivity polymer, found that the pulse thermography quantification method derived by Ringermacher et al. could also be used with longer pulses when the starting point for the peak slope time was taken as the midpoint of the pulse.

Figure 2 shows how equation 2 is applied to the temperature contrast $(\Delta \mathrm{T})$ with the starting point for the peak slope time determination at half the pulse length. The peak temperature contrast time for the defect $\left(t_{d}\right)$ is found by fitting a polynomial to the temperature contrast starting at the end of the pulse. The $t_{d}$ is added to half of the pulse length to find $\left(t_{s}\right)$ the actual peak slope time $\left(t_{s}\right)$ used for calculation of the defect depth. The updated equation can be seen as:

$$
t_{d}+\frac{1}{2} t_{p}=t_{s}=\frac{3.64 L^{2}}{\pi^{2} \alpha}
$$

When the pulse length is small relative to the peak slope time $\left(t_{p}<<t_{s}\right)$, the original equation for quantifying defect depths via peak slope contrast time is recovered.

The focus of this paper is to analyze the limitations of using longer pulse lengths to accurately quantify defect depths while still utilizing the depth/time correlations from the surface temperature decay equation. With this approach, multiple quantification methods can still be utilized for defect detection. This method based on heating with longer pulses could allow for possible implementation into online quality monitoring for additive manufacturing processes. The impact of pulse length was studied numerically in both polymers and metals using the peak

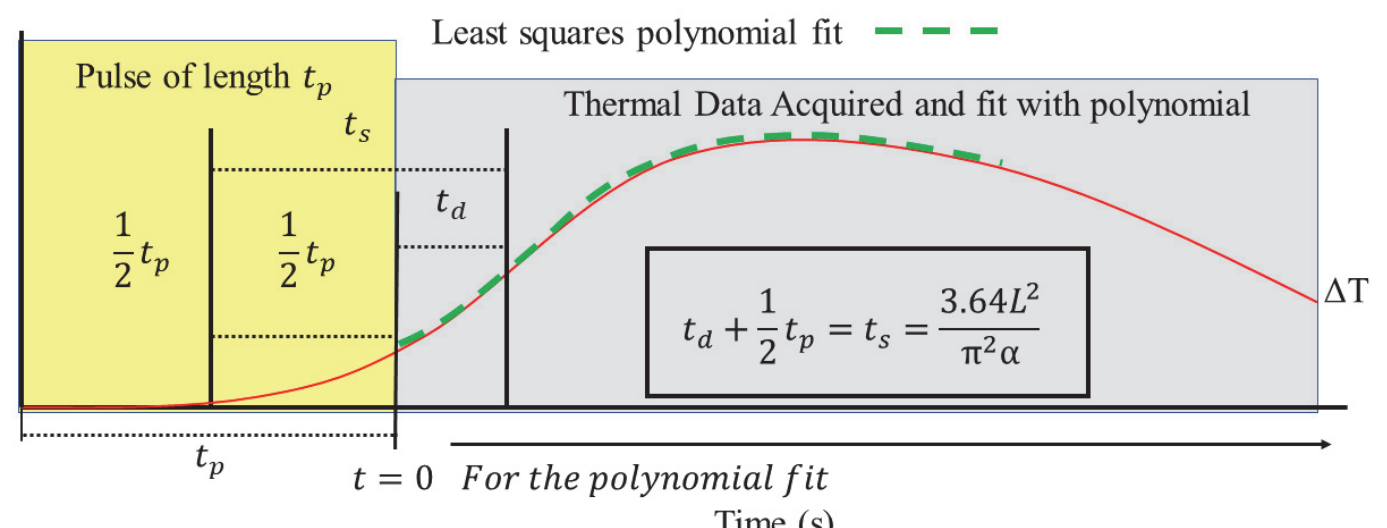

Time (s)

Figure 2 Schematic representation of time measurements in defect-depth calculation. $\Delta \mathrm{T}$ represents the temperature contrast between defective and sound regions. The peak-slope is calculated from a polynomial fit of the data after the end of the pulse, but the peak slope time $\left(t_{s}\right)$ used for defect-depth calculation includes half the pulse length $\left(t_{p} / 2\right)$. 

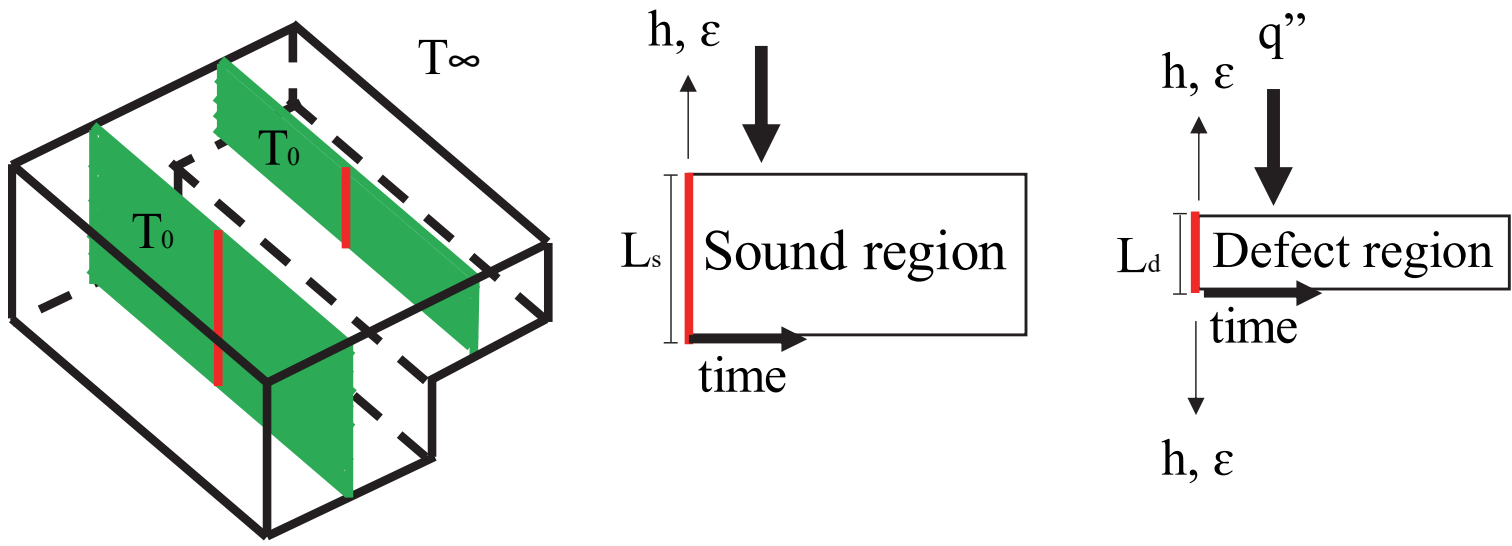

Figure 3 Schematic representation of the simulation boundary conditions used for 1D simulation of pulse thermographic method. (Left) A 3D case is simplified to two 1D models to represent a defect free region (middle) and defect region (right). Convection with a coefficient of $h=10 \mathrm{~W} \mathrm{~m}^{-2} \mathrm{~K}$ and radiation with emissivity $(\varepsilon=0.9)$ were applied at the top of both and backside of the defect. The initial and atmospheric temperature $\left(T_{0}, T_{\infty}\right)=$ 298K. $L_{d}$ is the defect depth and $L_{s}$ is the depth of the sound region of the specimen.

temperature contrast using 1D transient thermal models. These results were then validated using experimental measurements for the peak temperature contrast method in two 3D printed polymers. The $\log 2^{\text {nd }}$ derivative method shows similar trends with pulse length but is not reported here in the interest of space.

\section{Simulation Approach}

The method of Pulse Thermography (PT) is based on 1D heat conduction into the part from the surface. Therefore, the simulation was done with two separate 1D models to represent the defective and sound regions as seen in Figure 3. The simulation includes both the full duration of the applied heat pulse and a period of time sufficient to extract the significant information. The specific time was varied based on thermal properties of the material being studied. Upon calculation of the internal temperature distribution over time, predicted surface temperature over time is extracted for both the defective region simulation and the sound region simulation.

The defective region has a thickness equal to the defect depth $\left(\mathrm{L}_{d}\right.$ in Figure 3$)$ while the sound region is modeled with a thickness greater than twice the thickness of the defect $\left(\mathrm{L}_{\mathrm{s}}\right.$ in Figure 3 ). The sound region needs to be at a minimum of twice the thickness of the defect depth for equation 2 to accurately calculate defect depth with the constant 3.64 [6]. If the depth of the sound region is not more than twice the depth of the defect being measured the constant of 3.64 will need to be changed.

The sides are set as insulated boundary conditions based on the 1D conduction analysis and the magnitude of the heat flux was applied as a constant value over the pulse length. While the magnitude of the heat flux was held constant the pulse length was varied, thus varying the energy input. The heat flux applied during each pulse length was set at $4000 \mathrm{~W}$. The emissivity value of 0.9 was chosen to increase the effect of radiative heat loss as well as to better represent practical experimentation cases as most low-emissivity materials are coated with a thin layer of high absorptivity paint for testing. 
The 1D heat equation derived by Parker et al [1] neglects all heat losses. The assumption is acceptable when the measurements are taken in a short time period so that very little cooling takes place before the features of the defect are observed in the surface temperatures. However, with deeper defects or small thermal diffusivities, the peak times occur much further from the end of the pulse. As the cooling time increases, there is an increased possibility for the thermal losses to affect the measurements. To understand the effect heat losses have on the defect depth calculation, simulations were run with and without convective and radiative heat losses on both surfaces of the part and the defect with parameters selected to be representative of testing in an environment with a uniform initial temperature (Figure 3)Error! Reference source not found..

The Forward Time Center Space (FTCS) method was used to perform the 1D numerical solution. The FTCS is an approximation method derived from the 1D heat equation:

$$
\frac{\partial T}{\partial t}=\alpha \frac{\partial^{2} T}{\partial x^{2}}, \quad 0<x<L, \quad t \geq 0
$$

where $\alpha$ is the thermal diffusivity of the material and $\mathrm{L}$ is the thickness of the part. For derivation of the spatial and time approximation for the FTCS method see [13]. According to [14], for a stable solution the ratio $r$ must be less than 0.5 , i.e.:

$$
r=\frac{\alpha \Delta t}{\Delta x^{2}}<\frac{1}{2}
$$

For the simulations, the time discretization was varied based on the material being simulated to assure convergence. The simulation was implemented in MATLAB with defect depth calculation by peak temperature contrast method. Solutions were verified against a commercial thermal solver with the same boundary conditions, and there were minimal differences between the two methods attributed to different time and spatial discretization between the two approaches. To more accurately simulate experimental measurements of the peak slope time defect depth quantification, a normally distributed random noise with a standard deviation of $0.0014 \mathrm{~K}$ was added to the simulation data. This was observed to have an impact on the minimum energy input required to solve for the defect depth, but if input energy is increased there is not a significant impact of the noise on the calculated depth values.

\section{Experimental Setup}

A FLIR SC4000 MWIR infrared camera (3-5 $\mu \mathrm{m}$ sensitivity) was used for experimental analysis of the surface temperature with a $50 \mathrm{~mm}$ indium antimonide ( $\mathrm{InSb}$ ) lens using the setup illustrated in Figure 4. The frame rate was set at $100 \mathrm{~Hz}$ with a focal plane array of 320x256 pixels. For pulse heating of the surface, two $500 \mathrm{~W}$ halogen bulbs were set approximately 7 in from the surface of the part with an incidence angle of less than 25 degrees. Two pieces of acrylic (PMMA) 


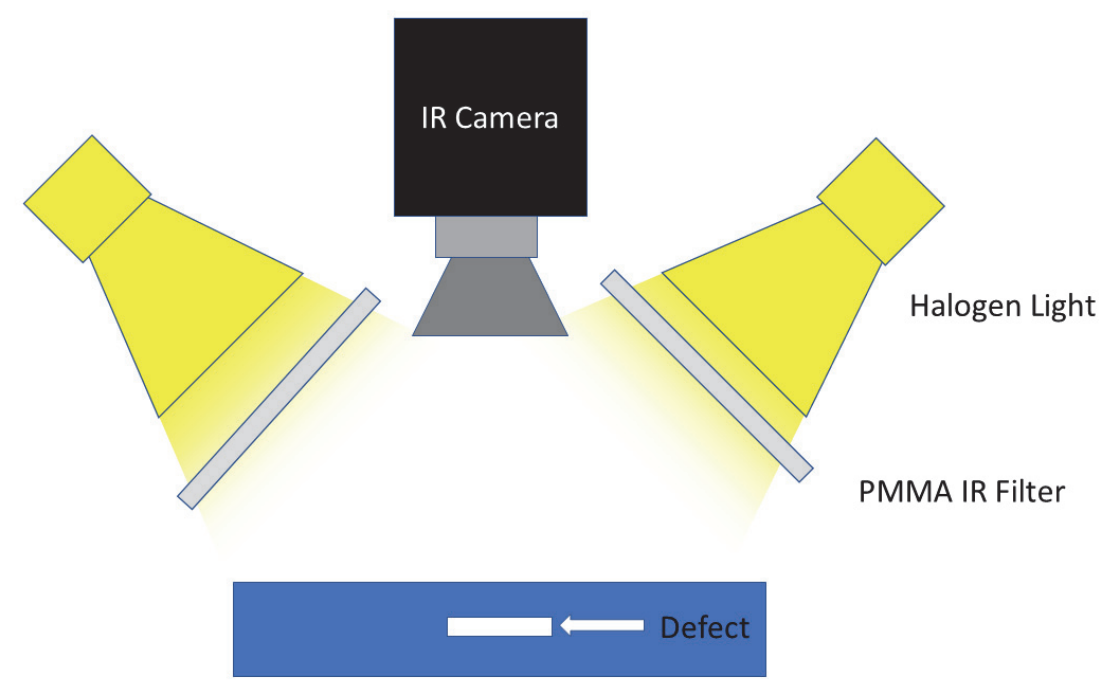

Figure 4 Diagram of experimental measurement system.

were used to filter infrared light to avoid temperature errors from reflections into the IR camera. The standard deviation of an equilibrium surface of a single pixel was approximately $0.05 \mathrm{~K}$. In this work, an array of pixels in each frame was averaged to reduce the measurement noise. When averaging over 840 pixels (ABS) and 2150 pixels (PLA), the standard deviation in temperature decreased to $0.007 \mathrm{~K}$ and $0.003 \mathrm{~K}$ respectively. This represents a spatial average over an $8 \mathrm{~mm} \mathrm{x}$ $8 \mathrm{~mm}$ and a $12 \mathrm{~mm} \times 12 \mathrm{~mm}$ area (PLA) respectively.

Table 1 Material properties of the four materials used in the defect depth simulation [12].

\begin{tabular}{|c|c|c|c|c|}
\hline Material & $\begin{array}{c}\text { Thermal } \\
\text { Conductivity, } \mathrm{K} \\
\left(\mathrm{Wm}^{-1} \mathrm{~K}^{-1}\right)\end{array}$ & $\begin{array}{c}\text { Specific Heat } \\
\text { Capacity, } \mathrm{C} \\
\left(\mathrm{Jkg}^{-1} \mathrm{~K}^{-1}\right)\end{array}$ & Density, $\rho$ & $\begin{array}{c}\text { Thermal } \\
\text { Diffusivity, } \alpha \\
\left(\mathrm{kg} \mathrm{m}^{-3}\right)\end{array}$ \\
\hline ABS & 0.2256 & 1386 & 1020 & $1.596 \times 10^{-7}$ \\
\hline PLA & 0.13 & 1800 & 1300 & $5.556 \times 10^{-8}$ \\
\hline Copper & 400 & 398 & 8912 & $1.128 \times 10^{-4}$ \\
\hline $316 \mathrm{SS}$ & 16.2 & 500 & 7990 & $4.055 \times 10^{-6}$ \\
\hline
\end{tabular}

Table 1 shows the material properties of the materials used in the simulation. For the experimental evaluation, the thermal diffusivity of acrylonitrile butadiene styrene (ABS) $(1.137 \mathrm{e}-$ $\left.07 \mathrm{~m}^{2} \mathrm{~s}^{-1}\right)$ and polylactic acid (PLA) $\left(1.414 \mathrm{e}-07 \mathrm{~m}^{2} \mathrm{~s}^{-1}\right)$ were used. These thermal diffusivity values were found experimentally for 3D-printed parts using the long pulse thermography method by Pierce and Crane [12], with pulse lengths of less than 500ms. This measured thermal diffusivity is below bulk values, likely due to the porosity and increased thermal resistance of the 3D printing process. Two defect depths were analyzed for both ABS and PLA. For ABS, the defect depths were $0.85 \mathrm{~mm}$ and $1.15 \mathrm{~mm}$ and the defect widths for each were $8 \times 8 \mathrm{~mm}$. For the PLA analysis, the 
two defect depths were $0.68 \mathrm{~mm}$ and $1.0 \mathrm{~mm}$ with a width of $12 \times 12 \mathrm{~mm}$. Each part was flashed with pulse lengths ranging from $0.25 \mathrm{~s}$ up to $5.0 \mathrm{~s}$. This range was chosen based on the measured values of thermal diffusivity for each material and their defect depths.

To acquire the peak slope times, a polynomial fit of the temperature contrast is performed to minimize the effect of the noise when taking the derivative as is commonly done in practice [4]. The zero-time point is taken as the midpoint of the pulse per Pierce and Crane [12]. The equation for the polynomial curve is then used to determine the derivative of the temperature contrast curve and the peak slope time is acquired from this derivative to find $t_{d}$ which is used to calculate $t_{s}$ per Eqn. 6.

\section{Results and Discussion}

The simulation results showed that heat loss from the surfaces (emissivity, convection) had negligible impact as these heat losses impacted calculated depths in the metals less than $1 \%$. While the lower thermal diffusivity and thus longer times before the peak slope occurs increased errors in polymers, the heat losses still only changed ABS and PLA depth calculations 1-2\%. This variation is much less than the possible variation and error from noise and fitting of the temperature data. While the difference was negligible, the simulations with heat loss were used in all the following results.

The simulation error is calculated by comparing the defect depth calculated from the simulation data to the actual defect depth. The error is analyzed as a function of the normalized pulse lengths defined in equation 4 and 5. As seen in Figure 5, the error in the defect depth calculation is well correlated to the normalized pulse length for both analysis methods and for both polymer and metals.

Figure 5 shows that for any given defect depth, the error in the defect calculation should stay below $5 \%$ if $t_{p} / t_{s}<1$. The maximum pulse length for a particular defect depth can be found by setting $t_{p} / t_{s}=1$ and substituting Eqn 2 so that

$$
t_{p}=\frac{3.64 L^{2}}{\pi^{2} \alpha}
$$

Under these conditions, the pulse length does not exceed the actual peak slope contrast time of a defect. For example, ABS with a $0.5 \mathrm{~mm}$ sub-surface defect, has a peak slope contrast time $\left(t_{s}\right)$ of $577 \mathrm{~ms}$. Thus, the maximum pulse length to accurately calculate this defect within $5 \%$ error is approximately the same at $577 \mathrm{~ms}$. The experimental long pulse results show the same trend of increasing error with longer pulse lengths as the simulations. In both cases, the error in the calculated depths trends upwards once the pulse length reaches approximately $70 \%$ of the actual peak slope contrast time for the material. Table 2 illustrates the maximum pulse length to obtain accurate defect detection within $5 \%$ error based on the simulation results for several materials and depths. 
Table 2 Predicted maximum pulse lengths for less than 5\% error for varying materials and defect depths

\begin{tabular}{|c|c|c|c|c|}
\hline $\begin{array}{c}\text { Defect } \\
\text { Depth }(\mathrm{mm})\end{array}$ & ABS & PLA & 316 SS & Copper \\
\cline { 2 - 5 } & Peak Slope & Peak Slope & Peak Slope & Peak Slope \\
\hline 0.3 & $0.21 \mathrm{~s}$ & $0.60 \mathrm{~s}$ & $0.008 \mathrm{~s}$ & $0.0003 \mathrm{~s}$ \\
\hline 0.5 & $0.58 \mathrm{~s}$ & $1.7 \mathrm{~s}$ & $0.023 \mathrm{~s}$ & $0.0008 \mathrm{~s}$ \\
\hline 1.0 & $2.3 \mathrm{~s}$ & $6.6 \mathrm{~s}$ & $0.091 \mathrm{~s}$ & $0.0033 \mathrm{~s}$ \\
\hline 2.0 & $9.2 \mathrm{~s}$ & $26 \mathrm{~s}$ & $0.36 \mathrm{~s}$ & $0.013 \mathrm{~s}$ \\
\hline
\end{tabular}

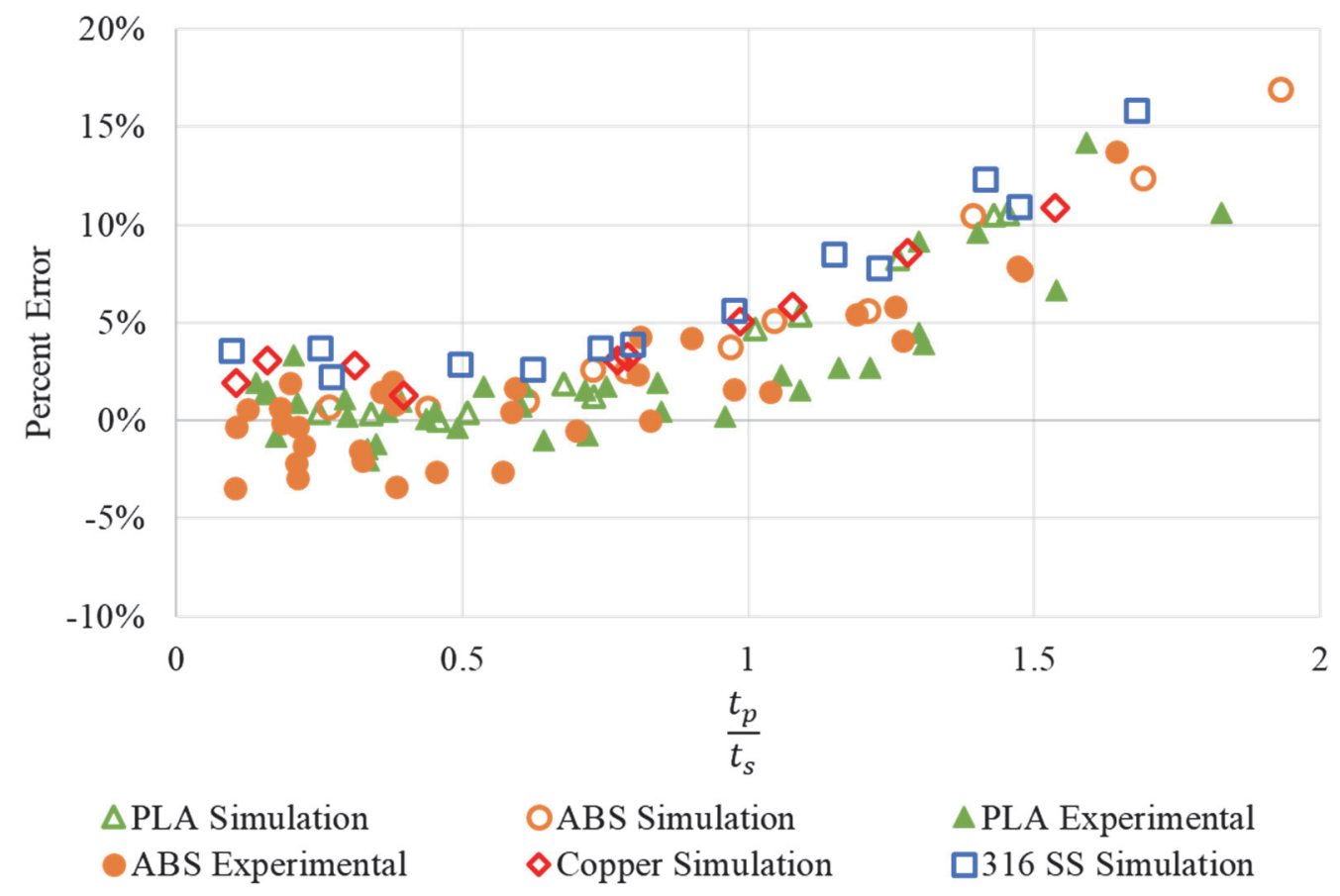

Figure 5 Comparison of defect depth calculations in simulation and experimental results for a range of materials.

The experimental results of ABS and PLA confirm the general trend of the percent error for defect depth calculation compared to the simulated calculations as seen in Figure 5. At approximately $75 \%$ of the characteristic peak slope times for each material and depth, the percent error in defect depth calculation begins to increase. As the normalized pulse length increases, the error in calculated depth increases but is only $15 \%$ at $t_{p} / t_{s}=2$.

Given the consistency of the error variation with pulse length, a correction factor can be applied similar to the method of Sun et al [6] in correcting for large defect depth to part thickness ratios. In this case, when the normalized pulse length exceeds approximately 0.75 , the error increases nearly linearly. Beyond the normalized pulse length of approximately 0.75 , the constant of 3.64 in Eqn 6 will need to be adjusted. The necessary adjustments to the constant can be seen in Figure 6. Using the $t_{d}$ from the experimental results of the ABS and PLA, the constants required 


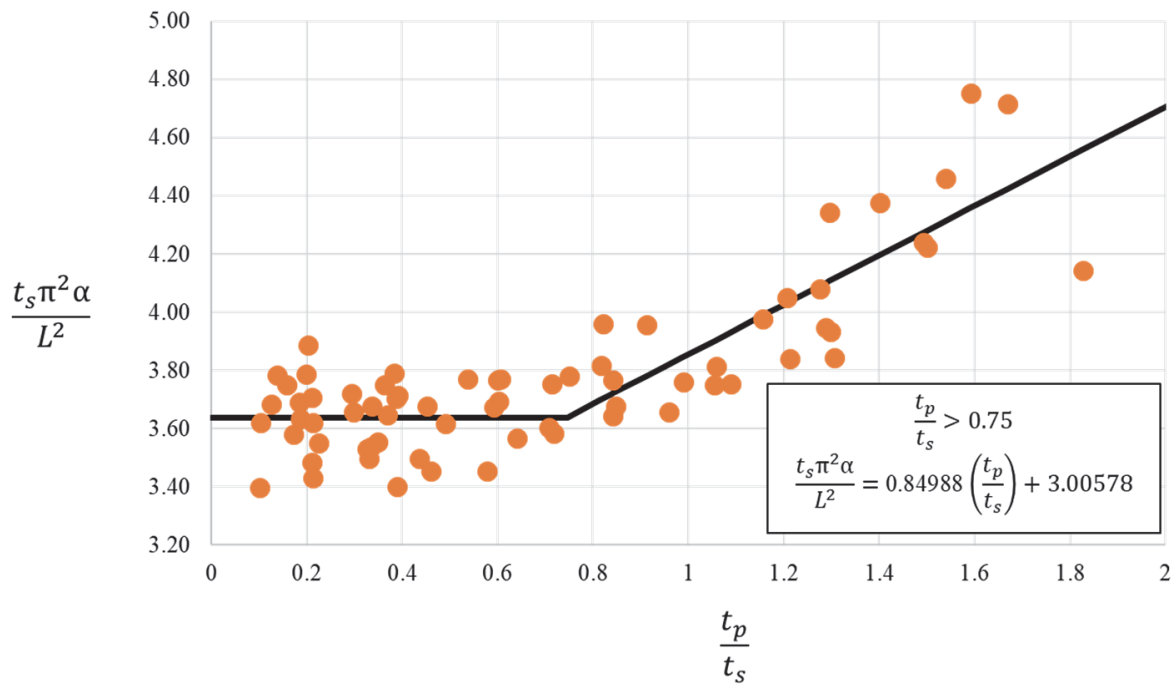

Figure 6 Experimental evaluations of the constants required to accurately calculate the exact defect depths of the ABS and PLA parts. The black line is the least squares fit of the experimental results.

to exactly calculate the depth were plotted in relation to $t_{p} / t_{s}$. A best fit of the data was applied and the best fit line should be used to accurately calculate the defect depth when $t_{p}$ is greater than approximately $75 \%$ of $t_{s}$. However, since even when $t_{p} / t_{s}$ is equal to 1 the experimental results showed accuracy within $5 \%$, the recommendation to re-evaluate the constant in equation 6 can be extended to $t_{p} / t_{s}$ ratios larger than 1 .

Figure 7 shows the experimental and simulation results with the constant in equation 6 reevaluated based on the respective normalized pulse length ratios for each trial. It can be seen that by re-evaluating the constants based on the normalized pulse length greater than 0.75 the calculated defect depths percent error dropped to within approximately 5\% of the actual depth across the entire range off experiments and simulation results.

Even after adjusting the constant based on the normalized pulse length, the method fails as $t_{p} / t_{s} \rightarrow 2$. The peak slope in the temperature contrast disappears after the normalized pulse length exceeds approximately 2 . As the pulse length increases for a constant defect depth, the time the peak slope occurs after the pulse $\left(t_{d}\right)$ will decrease to zero at $t_{p} / t_{s}=2$. Beyond this point, the continued heating alters the relationship and even before this limit, a short gap between the end of the pulse and the peak slope time complicates identification of the peak. Thus, a normalized pulse length of 2 is the maximum for accurately quantifying defect depths with a longer pulse using standard pulse thermography methods.

In comparing the experimental results to the simulation results, it can be seen that the percent error of the experimental results trended lower than the simulation for the same pulse length. This is likely due to the particular parameter range measured and/or the peculiarities of a $3 \mathrm{D}$ printed test sample. The variation may be due to effects such as nonuniform input flux or variation in the thermal properties due to small differences in the void content due to changes in the 3D printing processes used to create the parts. Even with $100 \%$ infill target, there will be a thermal resistance between each extruded filament and small voids. This could create a slightly lower thermal diffusivity that would offset the results. 


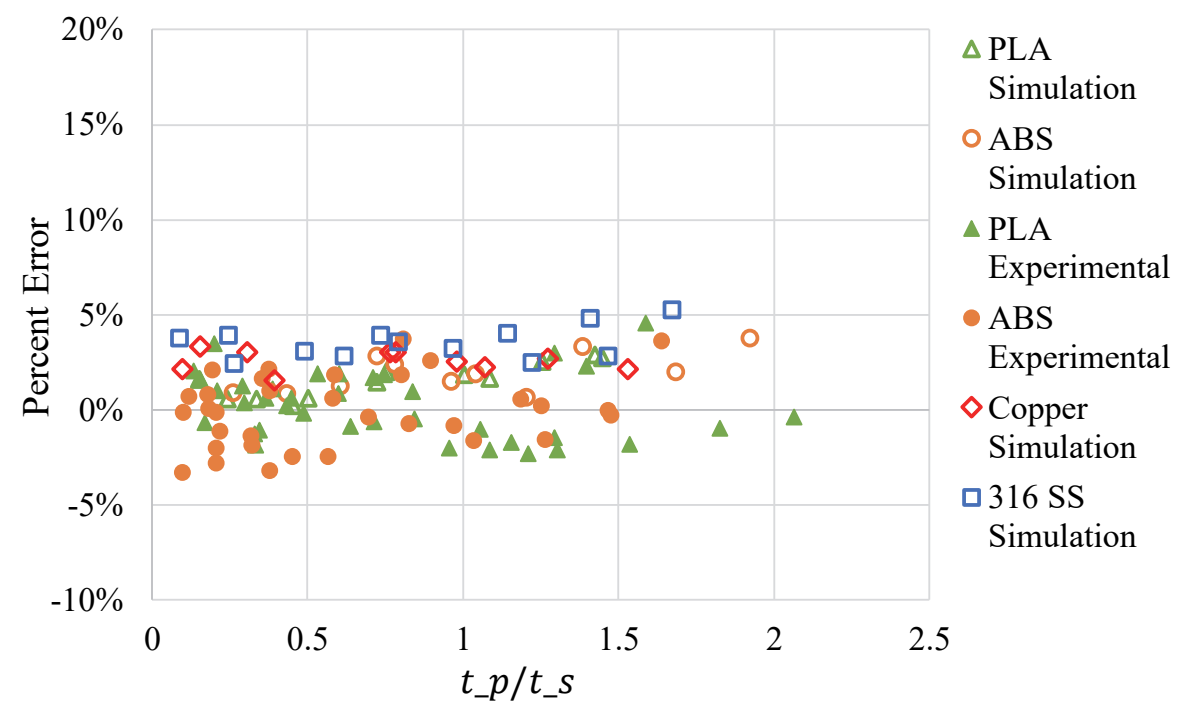

Figure 7 Comparison of defect depth calculations in simulation and experimental results after modifying the constants in equation 6 based on the normalized pulse length as described in Figure 5.

The ability to characterize the maximum allowable pulse length for accurate defect detection is a crucial benefit in analyzing a specific part. Based on the expected defect depths, a maximum pulse length can be selected that increases the energy input for higher signal to noise ratios. Comparing PLA versus ABS, two materials most commonly used for fused deposition modeling (FDM) printing, the thermal properties are different. At $4 \mathrm{~kW}$ power and a $2 \mathrm{~ms}$ pulse, the thermal contrast at the point of peak slope for ABS is $.002 \mathrm{~K}$ and PLA is $.001 \mathrm{~K}$. Both are below the expected experimental noise level of the SC4000 camera $\left(0.007^{\circ} \mathrm{C}\right.$ when averaged over a small region). Thus, either a longer pulse or more power to produce a larger energy input is required to quantify the defect depth.

As seen in Figure 8a, four different pulse lengths were compared producing the same overall energy input of $400 \mathrm{~J}$ into the part. For the case of the $2 \mathrm{~ms}$ pulse, the temperature increase of the surface is approximately $17^{\circ} \mathrm{C}$. This level of temperature increase could introduce more error due to possible phase changes and significantly added radiative heat losses. With a $320 \mathrm{~ms}$ pulse, the surface temperature doesn't exceed $2^{\circ} \mathrm{C}$, minimizing the effects of radiative losses and eliminating any possibility of phase changes in the part. It can be seen in Figure $8 \mathrm{~b}$, that since the overall energy input into the part is the same for the four different pulse rates, the temperature contrast between the defective and sound region is the same. Thus, to achieve the best signal to noise ratio and an increase possibility of accuracy in quantifying defect depth, increasing the pulse length is the better of the two choices.

\section{Conclusion}

Acceptable pulse length is a function of the depth of defects being examined and the thermal properties of the material. Both the simulation and the experimental results showed that the maximum pulse length achievable for a given material to be within 5\% error is equal to the peak slope time for the specific depth that is being measured. The experimental results trend on the lower side of the calculated error, allowing for pulse lengths up to 1.2 times the peak slope 

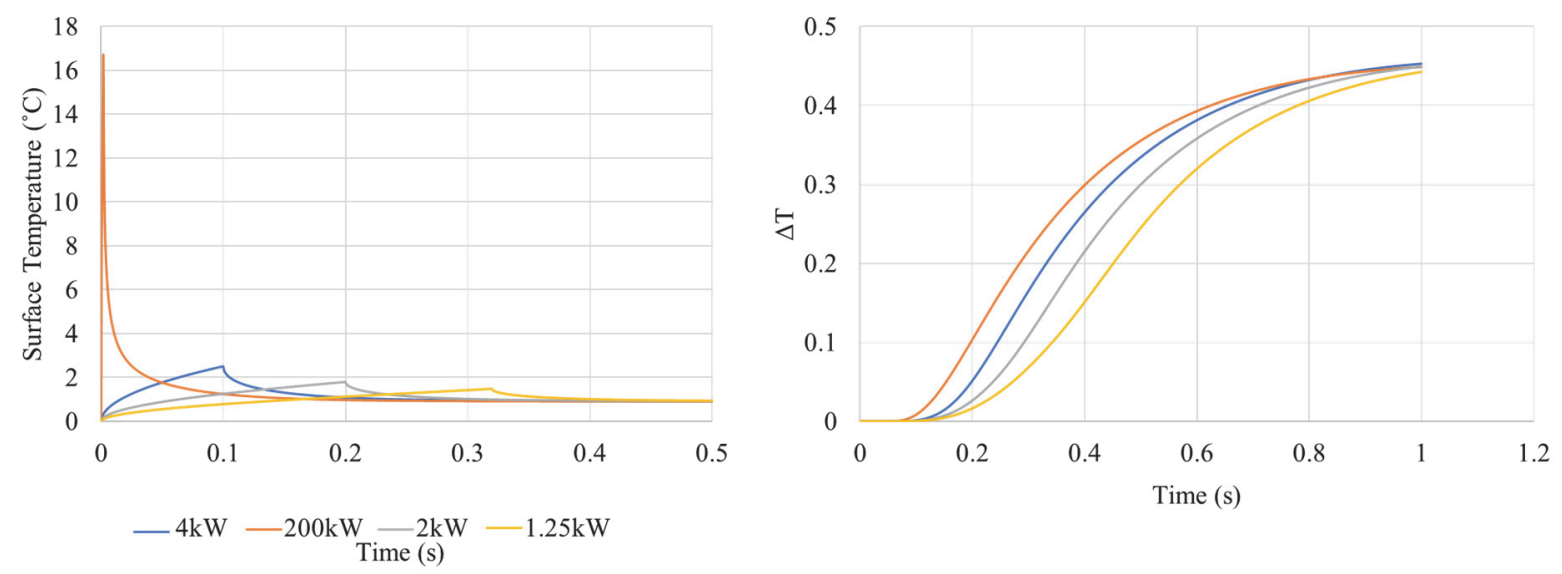

Figure 8 Simulation of Thermal Response to different pulse lengths but with constant energy. (Left) Comparison of temperature contrast produced by a $0.3 \mathrm{~mm}$ defect in $\mathrm{ABS}$ as a function of pulse length. (Right) Comparison of the surface temperature increase with varying pulse lengths producing the same amount of energy.

time in this case. Given the consistency of the error across a range of materials, the depth formula could be adjusted when the normalized pulse length exceeds 0.75 to more accurately quantify the defect depth. By utilizing the maximum allowable pulse length for accurate defect detection, the energy input can be significantly increased, creating larger thermal contrasts on the surface. This in turn minimizes the possible error from the signal to noise ratio. It also allows for defect depth measurements of a much wider range of defect depths as well as a much wider range of materials.

\section{Acknowledgements}

This work is funded in part by the Florida High Tech Corridor.

\section{References}

[1] W. J. Parker, R. J. Jenkins, C. P. Butler, and G. L. Abbott, "Flash Method of Determining Thermal Diffusivity, Heat Capacity, and Thermal Conductivity," Journal of Applied Physics, vol. 32, no. 9, p. 1679, 09// 1961.

[2] T. Liang, W. Ren, G. Y. Tian, M. Elradi, and Y. Gao, "Low energy impact damage detection in CFRP using eddy current pulsed thermography," Composite Structures, vol. 143, pp. 352-361, 2016/05/20/ 2016.

[3] H. I. Ringermacher, R. J. Archacki, and W. A. Veronesi, "Nondestructive testing: transient depth thermography," ed: Google Patents, 1998.

[4] D. Sharath, M. Menaka, and B. Venkatraman, "Defect Characterization Using Pulsed Thermography," Journal of Nondestructive Evaluation, Report no. 2, p. 134, 2013.

[5] S. M. Shepard, J. R. Lhota, B. A. Rubadeux, D. Wang, and T. Ahmed, "Reconstruction and enhancement of active thermographic image sequences," Optical Engineering, vol. 42, no. 5, p. 1337, 05// 2003.

[6] J. G. Sun, "Analysis of pulsed thermography methods for defect depth prediction," Journal of Heat Transfer, no. 4, p. 329, 2006. 
[7] Z. Zhi, T. Ning, F. Lichun, and Z. Cunlin, "Specified value based defect depth prediction using pulsed thermography," Journal of Applied Physics, Report no. 2, p. 023112, 2012.

[8] S. Masamune and J. Smith, "Thermal conductivity of beds of spherical particles," Industrial \& Engineering Chemistry Fundamentals, vol. 2, no. 2, pp. 136-143, 1963.

[9] I. Gibson, D. Rosen, and B. Stucker, Additive manufacturing technologies : 3D printing, rapid prototyping, and direct digital manufacturing. New York, NY : Springer, 2015.

Second edition., 2015.

[10] J. W. Kim, K. W. Yun, and H. C. Jung, "Investigation of optimal thermal injection conditions and the capability of IR thermography for detecting wall-thinning defects in small-diameter piping components," Nuclear Engineering and Design, vol. 262, pp. 39-51, 2013/09/01/2013.

[11] D. P. Almond, S. L. Angioni, and S. G. Pickering, "Long pulse excitation thermographic non-destructive evaluation," NDT \& E International, vol. 87, pp. 7-14, 2017/04/01/ 2017.

[12] J. R. Pierce and N. B. Crane, "Defect Detection in Polymer Components via Long Pulse Thermography," Journal of Nondestructive Evaluation, Submitted 2018.

[13] G. W. Recktenwald, "Finite-difference approximations to the heat equation," Mechanical Engineering, vol. 10, pp. 1-27, 2004. 